Abdel-Malek, K. and Yeh, H. J., (2000) "Local Dexterity Analysis for Open

Kinematic Chains," Mechanism and Machine Theory, Vol. 35, pp. 131-154.

\title{
LOCAL DEXTERITY ANALYSIS FOR OPEN KINEMATIC CHAINS
}

\author{
K. A. ABDEL-MALEK AND H. J. YEH \\ Department of Mechanical Engineering and Center for Computer Aided Design, The University of \\ Iowa, Iowa City, IA 52242, U.S.A.
}

\begin{abstract}
Two methods for determining dexterity of manipulator arms are presented. A numerical method is illustrated for determining all possible orientations of the end-effector of an open kinematic chain based upon a row-rank deficiency of the sub-Jacobian of the kinematic constraint equations. This numerical method has been adapted from a theory for determining the accessible output set for planar and spatial manipulators. Boundaries of regions representing orientation solutions are mapped using continuation algorithms. One dimensional curves in three dimensional space are traced. Orientations of the manipulator end-effector at a point are studied (local dexterity). Only local dexterity solutions can be found. A second analytical method is also presented that determines the global dexterity of the system. This method relies upon determining all singularities of the system and identifying singular surfaces and singular curves. Regions of singular surfaces that are boundary are identified and intersected with a service sphere. This method, however, although analytical and accurate, has many limitations. The two methods are compared via implementation into a six degree of freedom manipulator.
\end{abstract}

\section{INTRODUCTION}

Workspaces of robotic manipulators are important characteristics associated with the kinematics of the underlying mechanism. Knowledge of the workspace allows a designer to better understand the manipulator's functionality. The notion of workspaces has been studied by a number of researchers in the past [1-5]. Analytical conditions were used to obtain the workspace by Tsai and Soni [6], Yang and Lee [7], Lai and Menq [8], Freudenstein and Primrose [9], and Spanos and Kohli [10]. Numerical methods were also used by Haug et al. [11]. The general four degree of freedom revolute manipulator has been studied by Cecarelli and Vinciquerra [12]. Workspace of parallel manipulators has also been studied $[13,4,14]$.

The dexterous workspace has been defined by Kumar and Waldron [15] as the subspace of the workspace within which a vector on the end-effector may assume all orientations. Because of joint limits and geometric constraints (e.g., at workspace boundaries), the dexterous workspace may not include all of the workspace. The subject of dexterity and orientability has also been studied by numerous researchers in the field [15-17].

This paper introduces two methods for determining dexterity. A numerical method based upon criteria for determining the accessible output set [11] was adapted to determine local dexterity. Local dexterity will be further clarified in the following section. Due to its inherent numerical formulation (using continuation methods), it will be shown that this method can only find local dexterity. It will also be shown that this numerical method is dependent upon the determination of a starting point on the onedimensional curve defining the region of dexterity (called the service region). Therefore, only individual regions can be traced. Another method for determining the workspace of manipulators presented by Abdel-Malek and Yeh [18] will be used to determine global dexterity. This method will be shown to be 
efficient in terms of determining service regions indicating dexterity. Difficulties encountered in both methods will be discussed.

\section{LOCAL DEXTERITY, PART I}

Dexterity, as used in the context of this paper, is all possible orientations for the end-effector at a specified point in the workspace. A sphere located at this point may be used to detect all penetrations of the end-effector through it. This sphere was called the service sphere by Vinagradov [19]. The set of points on the sphere that are due to these penetrations are collectively called a service region which represents the local dexterity of a manipulator. At a specific point in the workspace, a service sphere may have a number of service regions. Determining all service regions means addressing the global dexterity of the system at a point.

For example, consider the n-degree-of-freedom manipulator shown in Fig. 1a. At a point $\mathbf{p}=\left[\begin{array}{lll}x^{*} & y^{*} & z^{*}\end{array}\right]^{T}$ in the workspace, the service sphere is located with radius equal to the dimension of the nth link. The vector joining the point $\mathbf{g}$ with $\mathbf{p}$ defines the orientation of the end-effector. Thus, the regions that $\mathbf{g}$ assumes on the surface of the sphere provide an indication of the dexterity of the manipulator at $\mathbf{p}$.

The first formulation is aimed at determining the boundary curves to these service regions. For an assembled configuration, the generalized coordinates $\mathbf{q}=\left[q_{1}, \ldots, q_{n}\right]^{T}$ satisfy independent holonomic kinematic constraint equations of the form

$$
\Phi^{P}\left[\begin{array}{c}
P_{x}(\mathbf{q})-x^{*} \\
P_{y}(\mathbf{q})-y^{*} \\
P_{y}(\mathbf{q})-z^{*}
\end{array}\right]=\mathbf{0}
$$

where $\left[\begin{array}{lll}P_{x}(\mathbf{q}) & P_{y}(\mathbf{q}) & P_{z}(\mathbf{q})\end{array}\right]^{T}$ is the position vector of the point $\mathbf{p}$ described using the DenavitHartenberg representation [20].

In order to include inequality constraints into the constraint equation, inequalities imposed on joint limits in the form of

are parametrized as

$$
q_{i}^{\mathrm{min}} \leq q_{i} \leq q_{i}^{\max }
$$

$$
q_{i}=a_{i}+b_{i} \sin \lambda_{i}
$$

where $a_{i}=\left(q_{i}{ }^{\max }+q_{i}{ }^{\min }\right) / 2$ and $b_{i}=\left(q_{i}{ }^{\max }-q_{i}{ }^{\min }\right) / 2$ are the mid point and half range of the inequality constraint. The vector of new generalized coordinates are $\lambda=\left[\lambda_{1}, \ldots, \lambda_{n}\right]^{T}$.

A service sphere at $\mathbf{p}$, parametrized in terms of output coordinates $\mathbf{u}=\left[\begin{array}{ll}\theta & \varphi\end{array}\right]^{T}$ can be written as

$$
\mathbf{x}_{\text {sphere }}(\mathbf{u})=\left[\begin{array}{c}
P_{x}(\mathbf{q})+r \cos \phi \cos \theta \\
P_{y}(\mathbf{q})+r \cos \phi \sin \theta \\
P_{z}(\mathbf{q})+r \sin \phi
\end{array}\right]
$$

where $r$ is the radius of the service sphere. The vector $\overline{\mathbf{p g}}$, where $\mathbf{g}=\left[\begin{array}{lll}g_{x} & g_{y} & g_{z}\end{array}\right]^{T}$ is a point chosen on the n-th link, can be written in terms of the spherical coordinates as 


$$
\left[\begin{array}{l}
g_{x}(\mathbf{q})-P_{x}(\mathbf{q}) \\
g_{y}(\mathbf{q})-P_{y}(\mathbf{q}) \\
g_{z}(\mathbf{q})-P_{z}(\mathbf{q})
\end{array}\right]=\left[\begin{array}{c}
r \cos \phi \cos \theta \\
r \cos \phi \sin \theta \\
r \sin \phi
\end{array}\right]
$$

The point $\mathbf{g}$ can exist on the surface of a sphere rotating with center at $\mathbf{p}$. Since $r$ is a known constant, only two of the three equations (Eq. 5) are needed to determine the spherical coordinates $\theta$ and $\phi$. Combining Eq. (11), two of Eqs. (5), the sphere angle constraints $\theta$ and $\phi$, and the parametrized joint limits, the vector equations of constraint are written as

$$
\Phi(\mu)=\left[\begin{array}{c}
P_{x}(\mathbf{q})-x^{*} \\
P_{y}(\mathbf{q})-y^{*} \\
P_{z}(\mathbf{q})-z^{*} \\
g_{x}(\mathbf{q})-P_{x}(\mathbf{q})-r \cos \phi \cos \theta \\
g_{y}(\mathbf{q})-P_{y}(\mathbf{q})-r \cos \phi \sin \theta \\
\theta-\left(\pi+\pi \sin \lambda_{1}\right) \\
\phi-\left(0+\frac{\pi}{2} \sin \lambda_{2}\right) \\
q_{1}-\left(a_{1}+b_{1} \sin \lambda_{3}\right) \\
q_{n}-\left(a_{n}+b_{n} \sin \lambda_{n}\right)
\end{array}\right]=\mathbf{0}
$$

where $\mu=\left[\begin{array}{lll}\mathbf{u}^{T} & \mathbf{q}^{T} & \lambda^{T}\end{array}\right]^{T}$. The constraint vector function $\Phi(\mu)$ has $5+n+2=(n+7)$ constraints. Define a service region on the sphere as

$$
\mathrm{A} \equiv\{\mathbf{u}: \Phi(\mu)=\mathbf{0}, \text { for some } \mathbf{q} \text { and } \lambda\}
$$

It has been shown $[11,17]$ that the boundary of the workspace (called the accessible output set) is characterized by a row-rank deficiency of the sub-Jacobian of the kinematic constraint $\Phi_{w}$. In the formulation presented in this paper, a similar criteria is used for the boundary curves of service regions. The boundary of the service region is defined as

$$
\partial \mathrm{A} \subset\left\{\mathbf{u} \in \mathrm{A}: \operatorname{Rank} \Phi_{\mathbf{w}}<(n+7), \text { for some } \mathbf{w} \text { with } \Phi(\mathbf{u}, \mathbf{w})=\mathbf{0}\right\}
$$

where $\mathbf{w}=\left[\begin{array}{ll}\mathbf{q} & \lambda\end{array}\right]^{T}$ is $n+n+2=(2 n+2)$ and the Jacobian of the constraint function at an assembled configuration $\mathbf{q}^{0}$ is the matrix

$$
\Phi_{\mathbf{w}}\left(\mathbf{q}^{0}\right)=\left[\frac{\partial \Phi_{i}}{\partial w_{j}}\left(\mathbf{q}^{0}\right)\right]
$$

The criteria for the boundary curve of the service region can be reduced to analytical form by noting that the matrix is row-rank deficient if and only if its rows are linearly dependent [11]. This can be done by searching for a solution for the vector $\gamma$ in $\Phi_{\mathrm{w}}^{\mathrm{T}} \gamma=\mathbf{0}$, where $\gamma$ has dimension $(n+7)$. A necessary condition for determining the boundary of the service region is

$$
\partial \mathrm{A} \subset\left\{\mathbf{u} \in \mathrm{A}: \Phi_{\mathbf{w}}^{\mathrm{T}} \gamma=\mathbf{0}, \gamma^{\mathrm{T}} \gamma=1, \Phi(\mathbf{u}, \mathbf{w})=\mathbf{0}\right\}
$$


To project the solution onto a nonlinear one degree of freedom system, the matrix $\mathbf{G}^{*}(\mathbf{x})$ is formed (Eqs. 6 and 10) such that a solution is computed for

$$
\mathbf{G}^{*}(\mathbf{x}) \equiv\left[\begin{array}{c}
\Phi(\mathbf{u}, \mathbf{w}) \\
\Phi_{\mathbf{w}}^{\mathrm{T}} \gamma \\
\gamma^{\mathrm{T}} \gamma-1
\end{array}\right]=\mathbf{0}
$$

where $\mathbf{x}=\left[\begin{array}{lll}\mathbf{u}^{T} & \mathbf{w}^{T} & \gamma^{T}\end{array}\right]^{T}$. The constraint vector function $\Phi(\mathbf{u}, \mathbf{w})$ has $(n+7)$ equations, $\Phi_{\mathbf{w}}^{\mathrm{T}} \boldsymbol{\gamma}$ has $(2 n+2)$ equations, and $\gamma^{\mathrm{T}} \gamma-1$ has 1 equation. The total number of equations is $(3 n+10)$. The dimension of the new vector of generalized coordinates $\mathrm{x}$ is $2+(2 n+2)+(n+7)=3 n+11$. Thus, this is a one degree-of-freedom system.

At an initial configuration having the end-effector point $\mathbf{p}$ located at the target position $\left[\begin{array}{lll}x^{*} & y^{*} & z^{*}\end{array}\right]^{T}$, the initial set of coordinates $\left[\begin{array}{lll}\mathbf{u}^{o} & \mathbf{q}^{o} & \lambda^{o}\end{array}\right]^{T}$ satisfying the constraints $\Phi(\mu)$ of Eq.(6) can be calculated. In this configuration, the wrist point $\mathbf{g}\left(\mathbf{q}^{o}\right)$ may be located inside the service region. To compute a point near the boundary, the point $\mathbf{g}$ is 'dragged' along a direction vector until it reaches the boundary. This dragging procedure can be achieved by iteratively increasing the coordinates $\theta$ or $\phi$ in small stepsizes and using the modified Newton-Raphson method with the Moore-Penrose pseudo inverse to obtain a corrected configuration. The pseudo inverse method is used because the dimension of the vector $\mu=\left[\begin{array}{lll}\mathbf{u}^{T} & \mathbf{q}^{T} & \lambda^{T}\end{array}\right]^{T}$ is $(2 n+4)$ which yields a nonsquare Jacobian matrix.

In order to numerically trace the solution curve of Eq. (11), it is first necessary to find a starting point on the curve. In order to obtain a solution to

$$
\Phi_{\mu} \Delta \mu=(-\Phi)
$$

the problem can be written as

$$
\Delta \mu=\Phi_{\mu}^{\mathrm{T}}\left(\Phi_{\mu} \Phi_{\mu}^{\mathrm{T}}\right)^{-1}(-\Phi)
$$

and computed as

$$
\mu^{i+1}=\mu^{i}+\Delta \mu^{i}
$$

where the coefficient $\Phi_{\mu}^{\mathrm{T}}\left(\Phi_{\mu} \Phi_{\mu}^{\mathrm{T}}\right)^{-1}$ is called the Moore-Penrose pseudo inverse [21]. Thus given an initial guess $\mathbf{q}^{1}$, the Moore-Penrose pseudo inverse computes a starting point $\mathbf{q}^{*}$ on the boundary curve. Once a point close to the boundary is computed, the boundary constraints $\mathbf{G}^{*}(\mathbf{x})$ and the new coordinates $\mathbf{x}$ with another modified Newton-Raphson iteration with pseudo inverse are used to determine a point $\mathbf{x}$ on the boundary.

The boundary of the service region is traced using an experimental numerical code (called PITCON) based on the continuation method presented by Rheinboldt [22]. This method can be written as an equation of constraint

$$
\mathbf{G}_{\mathbf{x}}(\mathbf{x}) \mathbf{h}(\mathbf{x})=\mathbf{0}
$$

where $\mathbf{h}(\mathbf{x})$ is uniquely defined by Eq. (15) and

$$
\mathbf{h}(\mathbf{x})^{T} \mathbf{h}(\mathbf{x})=1
$$




$$
\operatorname{Det}\left[\begin{array}{c}
\mathbf{G}_{\mathbf{w}}(\mathbf{w}) \\
\mathbf{h}(\mathbf{w})^{T}
\end{array}\right]>0
$$

It should be emphasized that only one boundary curve is traced for each starting point. Although we have been able to determine many starting points through trial and error, we have not been able to analytically determine all starting points. Neither have we been able to conclude the number of service regions.

\section{EXAMPLE USING PART I}

Consider the six degree-of-freedom manipulator depicted in Fig. (2), with the following geometry $d_{1}=50, d_{3}=10, d_{4}=25, d_{6}=10$, and $a_{2}=20$. The manipulator comprises six revolute joints. Using the Denavit-Hartenberg representation method [20], the $(4 \times 4)$ homogeneous transformation matrices are formulated for each joint as

$$
\begin{aligned}
{ }^{0} \mathbf{T}_{1} & =\left[\begin{array}{cccc}
\cos q_{1} & 0 & \sin q_{1} & 0 \\
\sin q_{1} & 0 & -\cos q_{1} & 0 \\
0 & 1 & 0 & 50 \\
0 & 0 & 0 & 1
\end{array}\right],{ }^{1} \mathbf{T}_{2}=\left[\begin{array}{cccc}
\cos q_{2} & 0 & -\sin q_{2} & 20 \cos q_{2} \\
\sin q_{2} & 0 & \cos q_{2} & 20 \sin q_{2} \\
0 & -1 & 0 & 0 \\
0 & 0 & 0 & 1
\end{array}\right] \\
{ }^{2} \mathbf{T}_{3} & =\left[\begin{array}{cccc}
\sin q_{3} & 0 & \cos q_{3} & 0 \\
-\cos q_{3} & 0 & \sin q_{3} & 0 \\
0 & -1 & 1 & 10 \\
0 & 0 & 0 & 1
\end{array}\right],{ }^{3} \mathbf{T}_{4}=\left[\begin{array}{cccc}
\cos q_{4} & 0 & \sin q_{4} & 0 \\
\sin q_{4} & 0 & -\cos q_{4} & 0 \\
0 & 1 & 0 & d_{4} \\
0 & 0 & 0 & 1
\end{array}\right] \\
{ }^{4} \mathbf{T}_{5} & =\left[\begin{array}{cccc}
\cos q_{5} & 0 & -\sin q_{5} & 0 \\
\sin q_{5} & 0 & \cos q_{5} & 0 \\
0 & -1 & 0 & 0 \\
0 & 0 & 0 & 1
\end{array}\right], \text { and }{ }^{5} \mathbf{T}_{6}=\left[\begin{array}{cccc}
\cos q_{6} & -\sin q_{6} & 0 & 0 \\
\sin q_{6} & \cos q_{6} & 0 & 0 \\
0 & 0 & 1 & d_{6} \\
0 & 0 & 0 & 1
\end{array}\right]
\end{aligned}
$$

The resultant transformation matrix relating the end-effector reference frame to the zeroth reference frame is

$$
{ }^{0} \mathbf{T}_{6}=\prod_{i=0}^{6}{ }^{i-1} \mathbf{T}_{i}=\left[\begin{array}{cc}
{ }^{0} \mathbf{R}_{6}(\mathbf{q}) & { }^{0} \mathbf{p}_{6}(\mathbf{q}) \\
\mathbf{0} & 1
\end{array}\right]
$$

where $\mathbf{p}(\mathbf{q})=\left[\begin{array}{lll}P_{x}(\mathbf{q}) & P_{y}(\mathbf{q}) & P_{z}(\mathbf{q})\end{array}\right]^{T}$. Similarly, the coordinates of the point $\mathbf{g}$ can be determined as

$$
{ }^{0} \mathbf{T}_{5}=\prod_{i=0}^{5}{ }^{i-1} \mathbf{T}_{i}=\left[\begin{array}{cc}
{ }^{0} \mathbf{R}_{5}(\mathbf{q}) & \mathbf{g}(\mathbf{q}) \\
\mathbf{0} & 1
\end{array}\right]
$$

To compare this method with that presented in the following section, inequality constraints imposed only on the first three joints will be considered. The following inequality constraints

$$
\begin{gathered}
-\frac{\pi}{4} \leq q_{1} \leq \frac{5 \pi}{4} \\
-\frac{\pi}{4} \leq q_{2} \leq \frac{\pi}{2} \\
0 \leq q_{3} \leq 2 \pi
\end{gathered}
$$

are parametrized according to Eq. (3) as 


$$
\begin{gathered}
q_{1}=b_{1}+c_{1} \sin \lambda_{1}=\frac{\pi}{2}+\frac{3 \pi}{4} \sin \lambda_{1} \\
q_{2}=b_{2}+c_{2} \sin \lambda_{2}=\frac{\pi}{8}+\frac{3 \pi}{8} \sin \lambda_{2} \\
q_{3}=b_{3}+c_{3} \sin \lambda_{3}=\pi+\pi \sin \lambda_{3}
\end{gathered}
$$

At a point $\mathbf{p}^{*}=\left[\begin{array}{lll}0 & -25 & 55\end{array}\right]^{T}$, a service sphere is located and the constraint equation is

$$
\Phi(\mathbf{u}, \mathbf{q}, \lambda)=\left[\begin{array}{c}
P_{x}(\mathbf{q})-0 \\
P_{y}(\mathbf{q})+25 \\
P_{z}(\mathbf{q})-55 \\
g_{x}(\mathbf{q})-P_{x}(\mathbf{q})-r \cos \phi \cos \theta \\
g_{y}(\mathbf{q})-P_{y}(\mathbf{q})-r \cos \phi \sin \theta \\
\theta-\left(\pi+\pi \sin \lambda_{1}\right) \\
\phi-\left(0+\frac{\pi}{2} \sin \lambda_{2}\right) \\
q_{1}-(\pi / 2)-(3 \pi / 4) \sin \lambda_{3} \\
q_{2}-(\pi / 8)-(3 \pi / 8) \sin \lambda_{4} \\
q_{3}-\pi-\pi \sin \lambda_{3}
\end{array}\right]=\mathbf{0}
$$

The boundary curve is traced in Fig. (3). This curve is the boundary to a single service region on the service sphere. The significance of this curve will become apparent in the following section. Singular bifurcation points may occur during tracing and the numerical code (PITCON) may stop. It is suggested that higher-order singularities exist at these points. This problem was not addressed in this work. First order singularities have been discussed by Abdel-Malek and Yeh [18] and a method for computing tangents at bifurcation points has been demonstrated.

\section{DETERMINING LOCAL DEXTERITY, PART II}

The method presented in this section will employ a singularity criteria of the Jacobian. Due to its analytical formulation, the method is only valid for manipulators having spherical wrists. The manipulator will be segmented into two parts. The first part comprises the first three links and the second part comprises the last three links. The workspace for each segment will be determined. Surfaces that are boundary to the workspace generated by a point on the first segment (called the wrist point) will be analytically determined.

At a point $\mathbf{p}^{*}$ in the workspace, the service sphere is located. The intersection of the service sphere with the boundary to the wrist workspace results in service regions. Service regions due to the second segment will be determined. For the first segment, the vector function describing the coordinates of a point on the third link (called the wrist point) can be written as

$$
\mathbf{x}(\mathbf{q})={ }^{0} \mathbf{R}_{3}(\mathbf{q})^{3} \mathbf{x}_{w}+{ }^{0} \mathbf{p}_{3}(\mathbf{q})
$$

where $\mathbf{q}=\left[\begin{array}{lll}q_{1} & q_{2} & q_{3}\end{array}\right]^{T},{ }^{0} \mathbf{R}_{3}$ is the $(3 \times 3)$ rotation matrix, ${ }^{3} \mathbf{x}_{w}$ is the vector describing the wrist point resolved in the reference frame of link 3 , and ${ }^{0} \mathbf{p}_{3}$ is the position vector determined from ${ }^{0} \mathbf{T}_{3}$ (Eq. 
8). At a certain position in space, the generalized coordinates satisfy independent holonomic kinematic constraint equations of the form

$$
\Lambda(\mathbf{q})=\mathbf{x}(\mathbf{q})-{ }^{0} \mathbf{R}_{3}(\mathbf{q})^{3} \mathbf{x}_{w}-{ }^{0} \mathbf{p}_{3}(\mathbf{q})=\mathbf{0}
$$

Method II is applicable to manipulators having up to 6 axes with revolute and prismatic joints.

Define a singularity $\mathbf{q}^{s}$ as the value of a generalized coordinate that causes the mechanism to lose at least one degree of mobility [23]. Singularities of mechanisms were defined [11] as the values that make the Jacobian singular. A Jacobian of the constraint function can be determined as

$$
\Lambda_{\mathbf{q}}(\mathbf{q})=\left[\frac{\partial \Lambda_{i}}{\partial \mathbf{q}_{j}}(\mathbf{q})\right]
$$

To take into consideration the effect of inequality constraints in the parametrized form

$$
q_{i}=a_{i}+b_{i} \sin \lambda_{i}
$$

The Jacobian with respect to the new generalized coordinates can be written as

$$
\Lambda_{\lambda}(\mathbf{q})=\left[\frac{\partial \Lambda_{i}}{\partial \mathbf{q}_{j}} \frac{\partial \mathbf{q}_{j}}{\partial \lambda_{k}}(\mathbf{q})\right]=\Lambda_{\mathbf{q}} \mathbf{q}_{\lambda}(\mathbf{q})
$$

Singularities are determined by equating the determinant of $\Lambda_{\lambda}(\mathbf{q})$ to zero such that

$$
F(\lambda)=\left|\Lambda_{\mathbf{q}} \mathbf{q}_{\lambda}\right|=\mathbf{0}
$$

Solutions to Eq. (32) are values for $\lambda$ that are substituted into Eq. (30) to obtain first-order singularities in terms of the generalized coordinates $\mathbf{q}$. These singularities will be denoted by $q_{j}^{s_{i}}$, where the subscript indicates the joint number and the superscript indicates the number of that singularity. For example, $q_{2}^{s_{3}}$ indicates the third singularity of the second joint.

Substituting the set of singularities into the wrist position vector (Eq. 27) yields a vector describing a parametrized surface in two variables.

$$
\mathbf{x}\left(q_{i}^{s_{j}} \mid u, v\right)={ }^{0} \mathbf{R}_{3}(u, v)^{3} \mathbf{x}_{w}+{ }^{0} \mathbf{p}_{3}(u, v)
$$

where the notation $\mathbf{x}\left(q_{i}^{s_{j}} \mid u, v\right)$ indicates the vector function $\mathbf{x}$ evaluated at the singularity $q_{i}^{s_{j}}$ and parametrized in terms of $u$ and $v$. Inequality constraints imposed on the joint variables $u$ and $v$ are

$$
u^{\min } \leq u \leq u^{\max } \text { and } v^{\min } \leq v \leq v^{\max }
$$

Singular surfaces defined by Eq. (33) may exist on the boundary to the wrist workspace, internal to it, or both. In order to determine regions of the surface that are boundary to the wrist workspace, singular surfaces are intersected. The intersection of two singular surfaces yields singular curves. Define singular curves as trajectories of the wrist point at which the mechanism loses two or more degrees of mobility. Since resulting singular surfaces may be complicated, the intersection cannot be determined analytically. Numerical methods are necessary to trace the intersection curve. The intersection problem may be considered as solving the system of seven non-linear equations with eight variables where the constraint function $\mathbf{H}(\zeta)$ is 


$$
\mathbf{H}(\zeta)=\left[\begin{array}{c}
\mathbf{x}_{1}\left(q_{j}^{s_{i}} \mid u, v\right)-\mathbf{x}_{2}\left(q_{m}^{s_{k}} \mid s, t\right) \\
u-\left[\left(u_{1}+u_{2}\right) / 2+\sin \left(\lambda_{1}\right)\left(u_{2}-u_{1}\right) / 2\right] \\
v-\left[\left(v_{1}+v_{2}\right) / 2+\sin \left(\lambda_{2}\right)\left(v_{2}-v_{1}\right) / 2\right] \\
s-\left[\left(s_{1}+s_{2}\right) / 2+\sin \left(\lambda_{3}\right)\left(s_{2}-s_{1}\right) / 2\right] \\
t-\left[\left(t_{1}+t_{2}\right) / 2+\sin \left(\lambda_{4}\right)\left(t_{2}-t_{1}\right) / 2\right]
\end{array}\right]=\mathbf{0}
$$

where $\zeta=\left[\begin{array}{llllllll}u & v & s & t & \lambda_{1} & \lambda_{2} & \lambda_{3} & \lambda_{4}\end{array}\right]^{T}$ and the inequality constraints associated with each surface were parametrized per Eq. (3). The Jacobian of the constraint function $\mathbf{H}(\zeta)$ for a certain configuration $\zeta^{1}$ is the $(3 \times 3)$ matrix

$$
\mathbf{H}_{\zeta}\left(\zeta^{1}\right)=\left[\frac{\partial \mathbf{H}_{i}}{\partial \zeta_{j}}\left(\zeta^{1}\right)\right]
$$

To satisfy the constraint equation, an algorithm similar to Newton-Raphson iteration method is employed, as follows:

$$
\mathbf{H}_{\zeta} \Delta \zeta=-\mathbf{H}
$$

The Moore-Penrose inverse is used again to determine a starting point $\zeta^{*}$ on the intersection curve.

\subsection{Determining the boundary to the wrist workspace}

Once a starting point is found, the intersection curve is traced along the tangent direction by using the so-called marching method [24]. The tangent vector at the starting point can be computed by calculating the cross product of two normal vectors. At a known point on the surface $\mathbf{x}_{1}\left(q_{i}^{s_{j}} \mid u, v\right)$, there are two linearly independent tangents which are the derivatives of the surface with respect to its parameters. Let $\mathbf{x}_{u}^{1}$ denote the derivative of $\mathbf{x}_{1}$ with respect to $u$ and $\mathbf{x}_{v}^{1}$ denote the derivative of $\mathbf{x}_{1}$ with respect to $v$. The cross product of these vectors result in a normal to the surface such that

$$
\mathbf{n}^{1}=\left(\mathbf{x}_{u}^{1} \times \mathbf{x}_{v}^{1}\right) /\left\|\mathbf{x}_{u}^{1} \times \mathbf{x}_{v}^{1}\right\|
$$

Similarly, the second surface has a normal such that

and the tangent $\tau$ is computed as

$$
\mathbf{n}^{2}=\left(\mathbf{x}_{s}^{2} \times \mathbf{x}_{t}^{2}\right) /\left\|\mathbf{x}_{s}^{2} \times \mathbf{x}_{t}^{2}\right\|
$$

The new step constraint equation can be written as

$$
\tau=\mathbf{n}^{1} \times \mathbf{n}^{2}
$$

$$
\left[\mathbf{x}^{1}(u, v)-\mathbf{x}^{o}\right] \cdot \tau-c=0
$$

where $\mathbf{x}^{o}$ is the computed point, and $c$ is the step size.

To determine other intersection branches, singular behavior along the curve is studied. Allgower and Georg [21] suggest monitoring the curve for a change in sign of the following determinant

$$
\operatorname{det}\left(\begin{array}{c}
\mathbf{H}_{\zeta}(\tau(\sigma)) \\
\tau(\sigma)
\end{array}\right)
$$

where $\sigma$ is the arc length. Thus, to recognize that a singularity has occurred, Eq. (42) is used with a diminishing step variable until the singular point is computed. To trace the second curve, it is necessary to compute the second tangent at this bifurcation point. For this reason, it is assumed that the surfaces are tangent at the bifurcation point such that 


$$
\mathbf{x}_{u}^{1} \times \mathbf{x}_{v}^{1} / / \mathbf{x}_{s}^{2} \times \mathbf{x}_{t}^{2} \neq \mathbf{0}
$$

Lucaks [25] showed that a branch of a curve can be parametrized in terms of its arc length $\sigma$ as

$$
\mathbf{r}(\sigma)=\mathbf{x}^{1}(u(\sigma), v(\sigma))=\mathbf{x}^{2}(s(\sigma), t(\sigma))
$$

Taking the derivative of both sides of Eq. (44) with respect to $\sigma$ yields the tangent vector

$$
\frac{\partial \mathbf{r}}{\partial \sigma}=\mathbf{x}_{u}^{1}(u)_{\sigma}+\mathbf{x}_{v}^{1}(v)_{\sigma}=\mathbf{x}_{s}^{2}(s)_{\sigma}+\mathbf{x}_{t}^{2}(t)_{\sigma}
$$

Multiplying vectorially by $\mathbf{x}_{q_{1}}^{1}$ we get

$$
\left(\mathbf{x}_{u}^{1} \times \mathbf{x}_{v}^{1}\right) \cdot(v)_{\sigma}=\left(\mathbf{x}_{u}^{1} \times \mathbf{x}_{s}^{2}\right) \cdot(s)_{\sigma}+\left(\mathbf{x}_{u}^{1} \times \mathbf{x}_{t}^{2}\right) \cdot(t)_{\sigma}
$$

Dividing by $\mathbf{x}_{u}^{1} \times \mathbf{x}_{v}^{1}$ yields

$$
\begin{aligned}
& (s)_{\sigma}=\alpha(u)_{\sigma}+\beta(v)_{\sigma} \\
& (t)_{\sigma}=\gamma(u)_{\sigma}+\delta(v)_{\sigma}
\end{aligned}
$$

where

$$
\alpha \delta-\beta \gamma \neq 0
$$

Derive both sides of Eqs. (45) and (46) again yields

$$
\begin{gathered}
\mathbf{x}_{u u}^{1}\left[(u)_{\sigma}\right]^{2}+2 \mathbf{x}_{u v}^{1}(u)_{\sigma}(v)_{\sigma}+\mathbf{x}_{v v}^{1}\left[(v)_{\sigma}\right]^{2}+\mathbf{x}_{u}^{1}(u)_{\sigma \sigma}+\mathbf{x}_{v}^{1}(v)_{\sigma \sigma}= \\
\mathbf{x}_{s s}^{2}\left[(s)_{\sigma}\right]^{2}+2 \mathbf{x}_{s t}^{2}(s)_{\sigma}(s)_{\sigma}+\mathbf{x}_{t t}^{2}\left[(t)_{\sigma}\right]^{2}+\mathbf{x}_{t}^{2}(t)_{\sigma \sigma}+\mathbf{x}_{t}^{2}(t)_{\sigma \sigma}
\end{gathered}
$$

Using the dot product of $\mathbf{n}$ in both sides of Eqs. (30) and (31), yields

$$
\begin{gathered}
<\mathbf{x}_{u u}^{1}, \mathbf{n}>\left[(u)_{\sigma}\right]^{2}+2<\mathbf{x}_{u u}^{1}, \mathbf{n}>(u)_{\sigma}(v)_{\sigma}+<\mathbf{x}_{v v}^{1}, \mathbf{n}>\left[(v)_{\sigma}\right]^{2}= \\
<\mathbf{x}_{s s}^{2}, \mathbf{n}>\left[(s)_{\sigma}\right]^{2}+2<\mathbf{x}_{s t}^{2}, \mathbf{n}>(s)_{\sigma}(t)_{\sigma}+<\mathbf{x}_{t t}^{2}, \mathbf{n}>\left[(t)_{\sigma}\right]^{2}
\end{gathered}
$$

Substituting Eq. (47) and Eq. (48) into Eq. (52) and Eq. (53) yields the following relation

$$
\theta\left[(u)_{\sigma}\right]^{2}+2 \mu(u)_{\sigma}(v)_{\sigma}+\kappa\left[(v)_{\sigma}\right]^{2}=0
$$

which is a quadratic form that can be written as

$$
\left[\begin{array}{ll}
(u)_{\sigma} & (v)_{\sigma}
\end{array}\right]\left[\begin{array}{ll}
\theta & \mu \\
\mu & \kappa
\end{array}\right]\left[\begin{array}{l}
(u)_{\sigma} \\
(v)_{\sigma}
\end{array}\right]=0
$$

The eigenvalues of the above system are computed as

$$
\begin{aligned}
& \lambda_{1}=\frac{\theta+\kappa}{2}+\sqrt{\left(\frac{\theta-\kappa}{2}\right)^{2}+\mu^{2}} \\
& \lambda_{2}=\frac{\theta+\kappa}{2}-\sqrt{\left(\frac{\theta-\kappa}{2}\right)^{2}+\mu^{2}}
\end{aligned}
$$

Therefore the solution in the neighborhood of a singular point depends on the definiteness of the quadratic form. Furthermore, the two vectors defining the tangent directions are the corresponding eigenvectors. Vectors in the tangent directions $\mathbf{v}_{1}$ and $\mathbf{v}_{2}$ are determined such that

$$
\begin{aligned}
& \tau_{1}=\sqrt{-\lambda_{2}} \mathbf{v}_{1}+\sqrt{\lambda_{1}} \mathbf{v}_{2} \\
& \tau_{2}=\sqrt{-\lambda_{2}} \mathbf{v}_{1}-\sqrt{\lambda_{1}} \mathbf{v}_{2}
\end{aligned}
$$

\subsection{Perturbation method}

It remains to determine whether these sub-surfaces are internal or boundary surfaces. This can be performed by perturbing a known point on the sub-surface and determining whether both perturbed points satisfy the constraint equation. Any point can be chosen, provided that it is not on the boundary 
of the sub-surface. For a sub-surface $\Psi^{i}(\zeta)$ having a known parametrization and a known boundary, the partial derivatives with respect to the parametrization variables $q_{1}$ and $q_{2}$ are $\partial \Psi^{i} / \partial q_{1}$ and $\partial \Psi^{i} / \partial q_{2}$. At any regular point $\mathbf{q}^{o}$ on the sub-surface, these vectors are linearly independent and tangent to the coordinate curves through $\mathbf{q}^{o}$ (they span the tangent plane of $\Psi^{i}(\mathbf{q})$ at $\mathbf{q}^{o}$ ). The unit vector $\hat{\mathbf{n}}$, which is orthogonal to those vectors, is

$$
\hat{\mathbf{n}}\left(\mathbf{q}^{o}\right)=\frac{\left(\frac{\partial \Psi^{i}}{\partial q_{1}} \times \frac{\partial \Psi^{i}}{\partial q_{2}}\right)}{\left\|\frac{\partial \Psi^{i}}{\partial q_{1}} \times \frac{\partial \Psi^{i}}{\partial q_{2}}\right\|}
$$

For a small perturbation $\partial \varepsilon$ about the point $\mathbf{q}^{o}$ and along the normal $\hat{\mathbf{n}}\left(\mathbf{q}^{o}\right)$, the coordinates of the perturbed points are

$$
\mathbf{x}_{1,2}\left(\mathbf{q}^{o}\right)=\Psi^{i}\left(\mathbf{q}^{o}\right) \pm \partial \hat{\varepsilon} \hat{\mathbf{n}}\left(\mathbf{q}^{o}\right)
$$

If the perturbed point is inside the wrist workspace, it has to satisfy the constraint equation (Eq. 28), subject to inequality constraints of Eq. (30). Substituting Eq. (61) into Eq. (28) and augmenting the system of equations with the parametrized inequality constraints, the perturbed point is inside the wrist workspace if there exists a solution to the following system of equations:

$$
\left[\begin{array}{c}
{ }^{0} \mathbf{R}_{3}{ }^{3} \mathbf{x}_{w}+{ }^{0} \mathbf{p}_{3}-\Psi^{i}\left(\mathbf{q}^{o}\right) \mp \partial \varepsilon \hat{\mathbf{n}}\left(\mathbf{q}^{o}\right) \\
q_{1}-b_{1}-c_{1} \sin \lambda_{1} \\
q_{2}-b_{2}-c_{2} \sin \lambda_{2} \\
q_{3}-b_{3}-c_{3} \sin \lambda_{3}
\end{array}\right]=\mathbf{0}
$$

Finding solutions to Eq. (62) indicates that the sub-surface is internal. Thus, a sub-surface $\Psi^{i}(\mathbf{q})$ is internal if and only if there exists a solution for Eq. (62) for both perturbations $\pm \partial \varepsilon$. Sub-surfaces that are boundary to the wrist workspace are used to construct the boundary to the wrist workspace.

\subsection{Determining service regions}

At a point $\mathbf{p}^{*}$ in the workspace, the service sphere is centered. The maximum distance between a point on the end-effector and the wrist point is assigned to be the radius of the service sphere. Therefore, the wrist point may assume position on the surface of the sphere.

Analytical boundaries to the wrist workspace have been determined in the foregoing discussion. The intersection of the service sphere with the boundary to the workspace yields regions that are accessible by the wrist point. These service regions are further restricted by joint limits imposed on the second segment of the manipulator. These limits have been discussed in recent work [18] and will not be discussed in this paper. Therefore, only inequality constraints associated with the first three joints will be considered. The service region $\operatorname{SR}_{i}(\mathbf{p}, \mathbf{q})$ is a set that exists on the surface of the service sphere such that

$$
S R_{i}(\mathbf{p}, \mathbf{q})=\left\{\mathbf{x}_{s s}(u, v)=\mathbf{0}, \text { for some } \mathbf{q}\right\}
$$

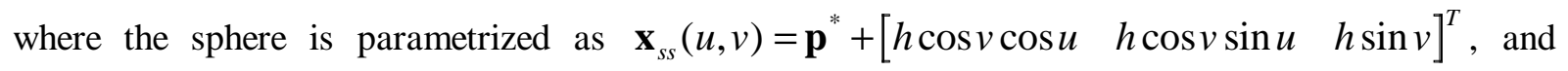
belongs to the wrist workspace such that

$$
S R_{i}(\mathbf{p}, \mathbf{q})=\{\Phi(\mathbf{q})=\mathbf{0}, \text { for some } \mathbf{q}\}
$$


Since the boundary of the wrist workspace has been analytically determined, it is possible to intersect each boundary sub-surface with the service sphere to determine curves that are boundary to service regions. To determine whether the service region is enclosed by the intersecting curve or lies outside it, a ray is cast in the direction of the normal passing through $\mathbf{p}$. The ray will intersect the sphere in two points $\mathbf{s}_{1}$ and $\mathbf{s}_{2}$. The service region is identified by determining which of these points satisfies the local constraint equation. A local constraint equation accounts for the inequality constraint associated with the singularity of the sub-surface. The intersection curve is the set of solutions to $\Lambda_{1}$, such that

$$
\Lambda_{1}=\left[\begin{array}{c}
\Psi^{i}(\mathbf{q})-\mathbf{x}_{s s}(u, v) \\
q_{i}-a_{i}-b_{i} \sin \lambda_{i}
\end{array}\right]=\mathbf{0}
$$

The service region is identified by determining one of the points on the ray satisfying the following

$$
\Lambda_{2}=\left[\begin{array}{c}
\Psi^{i}(\mathbf{q})+t \hat{\mathbf{n}}(\mathbf{p})-\mathbf{x}_{s s}(u, v) \\
q^{\text {local }}-a-b \sin \lambda
\end{array}\right]=\mathbf{0}
$$

Numerical solutions to Eq. (66) are obtained.

\section{EXAMPLE USING PART II}

Consider the six degree-of-freedom used in demonstrating part I of this paper (Fig. 1). Consider the first three joints as being segment number 1 responsible for locating the wrist point $\mathbf{w}$. Boundary surfaces to the wrist workspace for the wrist point at ${ }^{3} \mathbf{x}_{w}=\left[\begin{array}{lll}0 & 0 & d_{4}\end{array}\right]^{T}$ will be studied.

The vector function describing the position of the wrist point is

$$
\mathbf{x}(\mathbf{q})={ }^{0} \mathbf{x}_{3}=\left[\begin{array}{c}
10 \cos q_{1} \cos q_{2} \cos q_{3}-10 \sin q_{1} \sin q_{3}-10 \cos q_{1} \sin q_{2}+20 \cos q_{1} \cos q_{2} \\
10 \sin q_{1} \cos q_{2} \cos q_{3}+10 \cos q_{1} \sin q_{3}-10 \sin q_{1} \sin q_{2}+20 \sin q_{1} \cos q_{2} \\
10 \sin q_{2} \cos q_{3}+10 \cos q_{2}+20 \sin q_{2}+50
\end{array}\right]
$$

For a general point $\mathbf{x}=\left[\begin{array}{lll}x & y & z\end{array}\right]^{T}$ on link 3 of the manipulator, the independent holonomic constraint equations are

$$
\Lambda(\mathbf{q})=\left[\begin{array}{c}
x-10 \cos q_{1} \cos q_{2} \cos q_{3}+10 \sin q_{1} \sin q_{3}+10 \cos q_{1} \sin q_{2}-20 \cos q_{1} \cos q_{2} \\
y-10 \sin q_{1} \cos q_{2} \cos q_{3}-10 \cos q_{1} \sin q_{3}+10 \sin q_{1} \sin q_{2}-20 \sin q_{1} \cos q_{2} \\
z-10 \sin q_{2} \cos q_{3}-10 \cos q_{2}-20 \sin q_{2}-50
\end{array}\right]=\mathbf{0}
$$

subject to the following joint limits

$$
-\frac{\pi}{4} \leq q_{1} \leq \frac{5 \pi}{4},-\frac{\pi}{4} \leq q_{2} \leq \frac{\pi}{2}, \text { and } 0 \leq q_{3} \leq 2 \pi
$$

which are parametrized in Eqs. (23-25). To evaluate the Jacobian with respect to the new generalized coordinates $\lambda_{i}$, the matrices $\Lambda_{\mathbf{q}}$ and $\mathbf{q}_{\lambda}$ are evaluated such that

$$
\begin{gathered}
\Lambda_{\mathbf{q}}=\left[\begin{array}{cc}
-10 \sin q_{1} \cos q_{2} \cos q_{3}-10 \cos q_{1} \sin q_{3}+10 \sin q_{1} \sin q_{2}-20 \sin q_{1} \cos q_{2} \\
10 \cos q_{1} \cos q_{2} \cos q_{3}-10 \sin q_{1} \sin q_{3}-10 \cos q_{1} \sin q_{2}+20 \cos q_{1} \cos q_{2} \\
0 & -10 \cos q_{1} \cos q_{2} \sin q_{3}-10 \sin q_{1} \cos q_{3} \\
-10 \cos q_{1} \sin q_{2} \cos q_{3}-10 \cos q_{1} \cos q_{2}-20 \cos q_{1} \sin q_{2} & -10 \sin q_{1} \cos q_{2} \sin q_{3}+10 \cos q_{1} \cos q_{3} \\
-10 \sin q_{1} \sin q_{2} \cos q_{3}-10 \sin q_{1} \cos q_{2}-20 \sin q_{1} \sin q_{2} & -10 \sin q_{2} \sin q_{3} \\
10 \cos q_{2} \cos q_{3}-10 \sin q_{2}+20 \cos q_{2} &
\end{array}\right]
\end{gathered}
$$




\subsection{Computing singularities}

$$
\mathbf{q}_{\lambda}=\left[\begin{array}{ccc}
\frac{3 \pi}{4} \cos \lambda_{1} & 0 & 0 \\
0 & \frac{3 \pi}{8} \cos \lambda_{2} & 0 \\
0 & 0 & \pi \cos \lambda_{3}
\end{array}\right]
$$

Internal and boundary singularities are computed by evaluating the determinant of the Jacobian and equating to zero such that

$$
\begin{aligned}
& \left|\Lambda_{\mathbf{q}} \mathbf{q}_{\lambda}\right|=1125(-1)^{7 / 8} \cos \lambda_{1} \cos \lambda_{2} \cos \lambda_{3} \sin \left[\pi \sin \lambda_{3}\right] \\
& \quad+\left(-4+2(-1)^{1 / 2}\right)\left[(-1)^{1 / 4}+1\right] \cos \left(\frac{3 \pi \sin \lambda_{2}}{8}\right)+\left(1+(-1)^{1 / 4}\right) \cos \left(\frac{3 \sin \lambda_{2}-8 \sin \lambda_{3}}{8}\right) \\
& \quad+\left(1+(-1)^{1 / 4}\right) \cos \left(\frac{3 \pi \sin \lambda_{2}}{8}+\pi \sin \lambda_{3}\right)+\left(2+4(-1)^{1 / 2}\right)\left(1+(-1)^{1 / 4}\right) \sin \left(\frac{3 \pi \sin \lambda_{2}}{8}\right) \\
& \quad-\left((-1)^{1 / 2}-(-1)^{3 / 4}\right)\left[\sin \left(\frac{3 \sin \lambda_{2}-8 \sin \lambda_{3}}{8}\right)+\sin \left(\frac{3 \pi \sin \lambda_{2}}{8}+\pi \sin \lambda_{3}\right)\right]=0
\end{aligned}
$$

subject to the inequalities of Eq. (69). Singularities are determined by finding the roots of Eq. (72). For example, the term $\cos \lambda_{1}$ vanishes if $\lambda_{1}=\pi / 2$ or $\lambda_{1}=-\pi / 2$. Substituting $\lambda_{1}$ into Eq. (23) results in $q_{1}^{s_{1}}=-\frac{\pi}{4}$ or $q_{1}^{s_{2}}=\frac{5 \pi}{4}$. The other computed singularities are $q_{2}^{s_{3}}=-\frac{\pi}{4}, q_{2}^{s_{4}}=\frac{\pi}{2}$, $q_{3}^{s_{5}}=0,, q_{3}^{s_{6}}=2 \pi$, and $q_{3}^{s_{7}}=\pi$. The total number of first-order singularities is seven. The singularity $q_{3}^{s_{7}}=\pi$, however, is inside the interval $\left[\begin{array}{ll}0 & 2 \pi\end{array}\right]$. Since both of the endpoints are singularities, $q_{3}^{s_{7}}=\pi$ will not be considered. Singular surfaces are parametrized by substituting each singularity into Eq. (67). Note, however, that some of these surfaces are boundary to the workspace, other surfaces are internal, and some surfaces have parts that are on the boundary and parts that are internal. For example, substituting the singularity $q_{1}^{s_{1}}=-\frac{\pi}{4}$ into Eq. (67) yields the singular surface parametrized as follows

$$
\mathbf{x}^{1}\left(q_{1}^{s_{1}} \mid q_{2}, q_{3}\right)=\left[\begin{array}{c}
7.0711 \cos q_{2} \cos q_{3}+7.0711\left(\sin q_{3}-\sin q_{2}\right)+14.1421 \cos q_{2} \\
-7.0711 \cos q_{2} \cos q_{3}+7.0711\left(\sin q_{3}+\sin q_{2}\right)-14.1421 \cos q_{2} \\
10 \cos q_{3} \sin q_{2}+10 \cos q_{2}+20 \sin q_{2}+50
\end{array}\right]
$$

with inequality constraints defined as $0 \leq q_{3} \leq 2 \pi$ and $-\pi / 4 \leq q_{2} \leq \pi / 2$. Singular surface $\mathbf{x}^{1}\left(q_{1}^{s_{1}} \mid q_{2}, q_{3}\right)$ is shown in Fig. 5a.

Substituting the singularity $q_{1}^{s_{2}}=\frac{5 \pi}{4}$ into Eq. (67) yields the singular surface

$$
\mathbf{x}^{2}\left(q_{1}^{s_{2}} \mid q_{2}, q_{3}\right)=\left[\begin{array}{c}
-7.0711 \cos q_{3} \cos q_{2}+7.0711\left(\sin q_{3}+\sin q_{2}\right)-14.1421 \cos q_{2} \\
-7.0711 \cos q_{3} \cos q_{2}-7.0711\left(\sin q_{3}-\sin q_{2}\right)-14.1421 \cos q_{2} \\
10 \cos q_{3} \sin q_{2}+10 \cos q_{2}+20 \sin q_{2}+50
\end{array}\right]
$$


with inequality constraints as $0 \leq q_{3} \leq 2 \pi$ and $-\pi / 4 \leq q_{2} \leq \pi / 2$. The singular surface $\mathbf{x}^{2}\left(q_{1}^{s_{2}} \mid q_{2}, q_{3}\right)$ is shown in Fig. 5b. Singular surfaces due to the singularities $q_{3}^{s_{5}}=0, q_{3}^{s_{6}}=2 \pi$, $q_{2}^{s_{3}}=-\frac{\pi}{4}$, and $q_{2}^{s_{4}}=\frac{\pi}{2}$ are depicted in Fig. 6a, b, c, and d, respectively.

\subsection{Determining sub-surfaces}

Singular surfaces are divided into sub-surfaces by computing curves of intersection between them. Once these curves are determined and projected onto their respective parametric space of two variables, each region representing a sub-surface is studied for existence on the boundary of the workspace. To illustrate the determination of sub-surfaces, consider the intersection of the two surfaces $\mathbf{x}^{1}\left(q_{1}^{s_{1}} \mid q_{2}, q_{3}\right)=\mathbf{x}^{1}(u, v)$ and $\mathbf{x}^{2}\left(q_{1}^{s_{2}} \mid q_{2}, q_{3}\right)=\mathbf{x}^{2}(s, t)$, where the parameters have been replaced by $u, v, s$, and $t$ for simplicity. The constraint matrix (Eq. 35) can be written as

$$
\mathbf{H}(\zeta)=\left[\begin{array}{c}
\mathbf{x}^{1}(u, v)-\mathbf{x}^{2}(s, t) \\
u-\frac{\pi}{2}-\frac{3 \pi}{4} \sin \lambda_{1} \\
v-\frac{\pi}{8}-\frac{3 \pi}{8} \sin \lambda_{2} \\
s-\frac{\pi}{2}-\frac{3 \pi}{4} \sin \lambda_{3} \\
t-\frac{\pi}{8}-\frac{3 \pi}{8} \sin \lambda_{4}
\end{array}\right]=\mathbf{0}
$$

The starting point $\zeta^{*}$ computed using the Moore-Penrose pseudo inverse is

$\zeta^{*}=\left[\begin{array}{llllllll}0.6184 & 3.4086 & 0.6184 & 2.8745 & 0.1928 & 0.0851 & 0.1928 & -.0851\end{array}\right]$. Using $\zeta^{*}$ as a starting point, the algorithm for mapping marching curves is employed to continue tracing the curves. As the curve is being traced, the determinant of the sub-Jacobian changes signs and a bifurcation point is encountered at

$$
\zeta^{o}=\left[\begin{array}{llllllll}
0.7854 & 3.1416 & 0.7854 & 3.1416 & 0.3398 & 0.8306 \times 10^{-9} & 0.3398 & -0.8306 \times 10^{-9}
\end{array}\right] .
$$

The matrix of the quadratic equation (Eq. 35) is computed as

$$
\Lambda=\left[\begin{array}{cc}
\theta & \mu \\
\mu & \kappa
\end{array}\right]=\left[\begin{array}{cc}
14.1421 & 0.6830 \\
0.6830 & -28.2843
\end{array}\right]
$$

The eigenvalues of the quadratic equation are $\lambda_{1}=14.1421$ and $\lambda_{2}=-28.2843$. The first eigenvector due to the first eigenvalue is $\mathbf{v}_{1}=\left[\begin{array}{ll}1.0000 & 0.16099 \times 10^{-8}\end{array}\right]^{T}$, while the second eigenvector due to the second eigenvalue is $\mathbf{v}_{2}=\left[\begin{array}{lll}-0.16099 \times 10^{-8} & 1.0000\end{array}\right]^{T}$. To compute the tangents at the bifurcation point, a combination of the eigenvectors is used such that

$$
\begin{aligned}
& \tau^{1}=\sqrt{-\lambda_{2}} \mathbf{v}_{1}+\sqrt{\lambda_{1}} \mathbf{v}_{2}=\left[\begin{array}{ll}
5.3182 & -3.7606
\end{array}\right]^{T} \\
& \tau^{2}=\sqrt{-\lambda_{2}} \mathbf{v}_{1}-\sqrt{\lambda_{1}} \mathbf{v}_{2}=\left[\begin{array}{ll}
5.3182 & 3.7606
\end{array}\right]^{T}
\end{aligned}
$$

The normalized tangents computed in the parametric space at the bifurcation point are $\tau^{1}=\left[\begin{array}{ll}0.8165 & 0.5773\end{array}\right]^{T}$ and $\tau^{2}=\left[\begin{array}{lll}0.8165 & -0.5774\end{array}\right]^{T}$. The resulting intersecting curves in the parametric space are depicted in Fig. 7a, and depicted on the surfaces in Fig. 7b. 
In addition to the intersection curves resulting from the intersection between $\mathbf{x}^{1}\left(q_{1}^{s_{1}} \mid q_{2}, q_{3}\right)$ and $\mathbf{x}^{2}\left(q_{1}^{s_{2}} \mid q_{2}, q_{3}\right)$, surface $\mathbf{x}^{1}\left(q_{1}^{s_{1}} \mid q_{2}, q_{3}\right)$ intersects with other singular surfaces. The computed intersection curves due to other singular surfaces are superpositioned in Fig. 8. These four singular curves $\left(\mathbf{c}^{1}, \mathbf{c}^{2}, \mathbf{c}^{3}\right.$ and $\left.\mathbf{c}^{4}\right)$ partition surface $\mathbf{x}^{1}$ into twelve sub-surfaces.

To determine whether each sub-surface is a boundary or internal sub-surface to the wrist workspace, the perturbation method (Eq. 61) is implemented. For example, consider the point $p^{1}(\mathbf{q})$ on the subsurface $\Psi^{1}$ which has the set of generalized coordinates $q_{2}=0.4$ and $q_{3}=3.4$. Note that the subsurface $\Psi^{1}$ is defined as follows

$$
\Psi^{1}=\left[\begin{array}{c}
7.0711 \cos q_{2} \cos q_{3}+7.0711\left(\sin q_{3}-\sin q_{2}\right)+14.1421 \cos q_{2} \\
-7.0711 \cos q_{2} \cos q_{3}+7.0711\left(\sin q_{3}+\sin q_{3}\right)-14.1421 \cos q_{2} \\
10 \cos q_{2} \sin q_{3}+10 \cos q_{2}+20 \sin q_{2}+50
\end{array}\right]
$$

enclosed by the following boundary curve segments: $c^{1}$ on the interval $\left[n^{2} n^{8}\right]$, $c^{3}$ on the interval $\left[n^{8} n^{9}\right]$, and $c^{4}$ on the interval $\left[n^{9} n^{2}\right]$ (as illustrated in Fig. 8). To determine the normal to $\Psi^{1}$ using Eq. (60), partial derivatives representing tangent vectors are evaluated such that

and

$$
\frac{\partial \psi^{1}}{\partial q_{2}}=\left[\begin{array}{c}
-7.07 \sin q_{2} \cos q_{3}-7.07 \cos q_{2}-14.14 \sin q_{2} \\
7.07 \sin q_{2} \cos q_{3}+7.07 \cos q_{2}+14.14 \sin q_{2} \\
10 \cos q_{2} \cos q_{3}-10 \sin q_{2}+20 \cos q_{2}
\end{array}\right]
$$

The normal is computed

$$
\frac{\partial \psi^{1}}{\partial q_{3}}=\left[\begin{array}{c}
-7.07 \cos q_{2} \sin q_{3}+7.07 \cos q_{3} \\
7.07 \cos q_{2} \sin q_{3}+7.07 \cos q_{3} \\
-10 \sin q_{2} \sin q_{3}
\end{array}\right]
$$

$$
\begin{gathered}
\mathbf{n}=\frac{\partial \psi^{1}}{\partial q_{2}} \times \frac{\partial \psi^{1}}{\partial q_{3}}= \\
=\left[\begin{array}{c}
\frac{707}{-10}\left(\cos q_{2}\left(\cos q_{3}\right)^{2}-\sin q_{3} \cos q_{3}-\sin q_{3}+\sin q_{2} \cos q_{3}-2 \cos q_{2} \cos q_{3}\right) \\
\frac{707}{-10}\left(\cos q_{3} \sin q_{2}+\cos q_{2}\left(\cos q_{3}\right)^{2}-2 \sin q_{3}-\cos q_{3} \sin q_{3}+\cos q_{2} \cos q_{3}\right) \\
\frac{499849}{-5000}\left(\sin q_{2} \cos q_{3}-\cos q_{2} \cos q_{3}-\sin q_{2}\left(\cos q_{3}\right)^{2}\right)
\end{array}\right]
\end{gathered}
$$

The unit normal $\hat{\mathbf{n}}=\mathbf{n} /\|\mathbf{n}\|$ at the point $\mathbf{q}^{o}$ on the sub-surface $\Psi^{1}$ is evaluated

$$
\hat{\mathbf{n}}=\mathbf{n} /\|\mathbf{n}\|=\left[\begin{array}{lll}
0.098 & -0.452 & 0.887
\end{array}\right]^{T}
$$

For a small perturbation $\partial \varepsilon=+0.1$, the coordinates of the perturbed point are computed as

$$
\mathbf{x}_{1}=\Psi^{i}\left(\mathbf{q}^{o}\right)+\partial \varepsilon \hat{\mathbf{n}}\left(\mathbf{q}^{o}\right)=\left[\begin{array}{lll}
6.513 & -1.812 & 63.418
\end{array}\right]^{T}
$$


Forming Eq. (62) with $\mathbf{x}_{1}$ and the inequalities of Eq. (69), there exists a solution such that the generalized set is $\mathbf{q}=\left[\begin{array}{lll}2.321 & 1.222 & -3.502\end{array}\right]^{T}$. For a perturbation of $\partial \varepsilon=-0.1$, the coordinates of the perturbed point are

$$
\mathbf{x}_{2}=\Psi^{i}\left(\mathbf{q}^{o}\right)-\partial \hat{\varepsilon \mathbf{n}}\left(\mathbf{q}^{o}\right)=\left[\begin{array}{lll}
6.494 & -1.721 & 63.241
\end{array}\right]^{T}
$$

and the solution for this position is the generalized set $\mathbf{q}=\left[\begin{array}{llll}2.393 & 1.231 & -3.463\end{array}\right]^{T}$. Thus, both perturbation points are inside the wrist workspace which guarantees that this sub-surface is an internal one. For sub-surface $\Psi^{2}$, the point on this sub-surface is chosen as $p^{2}(\mathbf{q})$ which has coordinates $q_{2}=1.0$ and $q_{3}=4.4$. The unit normal $\hat{\mathbf{n}}=\mathbf{n} /\|\mathbf{n}\|$ at this point on the sub-surface $\Psi^{2}$ is evaluated as

$$
\hat{\mathbf{n}}=\mathbf{n} /\|\mathbf{n}\|=\left[\begin{array}{lll}
0.671 & 0.653 & 0.351
\end{array}\right]^{T}
$$

For a small perturbation $\partial \varepsilon=+0.1$, the coordinates of the perturbed point are

$$
\mathbf{x}_{1}=\Psi^{i}\left(\mathbf{q}^{o}\right)+\partial \hat{\mathbf{n}}\left(\mathbf{q}^{o}\right)=\left[\begin{array}{lll}
-6.211 & -7.245 & 69.646
\end{array}\right]^{T}
$$

For this point, a solution of Eq. (62) subject to inequality constraints of Eq. (69) can be found such that the set of generalized coordinates is $\mathbf{q}=\left[\begin{array}{lll}2.276 & 1.114 & 1.882\end{array}\right]^{T}$. A solution, however, cannot be found for $-\partial \varepsilon$. This indicates that $\Psi^{2}$ is a boundary sub-surface to the wrist workspace. Using this method, boundary sub-surfaces of each singular surface are determined. These surfaces are depicted in Fig. 9 . The volume enclosed by these surfaces is the wrist workspace. At the point $\mathbf{p}^{*}=\left[\begin{array}{lll}0 & -25 & 55\end{array}\right]^{T}$, the sphere is located and intersected with the boundary to the wrist workspace. Figure 10a depicts the sphere located at $\mathbf{p}^{*}$. The intersection of the sphere with the surfaces $\mathbf{x}^{1}\left(q_{1}^{s_{1}} \mid q_{2}, q_{3}\right)$ and $\mathbf{x}^{2}\left(q_{1}^{s_{2}} \mid q_{2}, q_{3}\right)$ yields two sets of boundary that essentially are the service regions (depicted in Fig. 10b).

\section{CONCLUSIONS}

Methods for determining dexterity have been presented. Method I is a numerical formulation that has been shown to work for any type of kinematic chain with various joint constraints. A vector constraint function is formulated to pose the problem in terms of a boundary curve to a service region. A service region was shown as being an indication of the local dexterity of the manipulator.

Method I was shown to be efficient in determining a point on the curve that is boundary to a service region within a few iterations. Numerical continuation methods were used to trace the boundary to the service region based upon a row rank deficiency of the sub-Jacobian of the constraint function. A deficiency of this method is in its inability to determine all service regions. Starting points computed using the Moore-Penrose pseudo inverse cannot be found for all curves. Difficulties were encountered in determining the number of existing service regions and therefore, the number of starting points required. The strength of this method is exhibited in its ability to account for all joint limits in a single formulation.

Method II formulates the problem in terms of two manipulator segments. An analytical method for determining the boundary to the workspace of the first segment was presented. It was shown that using this method, first-order singularities are computed by studying the Jacobian's singularity. It was also shown that surfaces where the manipulator looses one degree of freedom result from substituting these singularities into the constraint equation. Singular curves are then traced to partition singular surfaces 
into sub-surfaces. Sub-surfaces that are boundary to the wrist workspace are then used to form the analytical boundary. The service sphere is then intersected with the boundary to determine the service regions.

Because of the analytical results obtained in computing the wrist workspace, it is shown that all service regions are computed. Thus, the strength of method II are evident in determining the number of service regions existing on the service sphere. Joint limits imposed on the second segment of the manipulator, however, are more difficult to address.

\section{REFERENCES}

1. B. Roth, NBS Special Publications, 459, (1975).

2. A. Kumar, and K.J. Waldron, ASME Paper No. 80-DET, (1980).

3. V. Kumar, 1985, Masters Thesis, The Ohio State University, (1985)

4. C. Gosselin and J. Angeles, IEEE Trans. on Rob. and Auto., 6, (1990)

5. D.M. Emiris, Mechanisms and Machine Theory, 28, (1993)

6. Y.C. Tsai and A.H. Soni, J. of Mech. Design, 103, (1981).

7. D.C.H. Yang and T.W. Lee, J. of Mech., Trans. and Auto. Design, 105 (1983).

8. Z.C. Lai and A. Menq, IEEE J. Robotics and Automation, 4, (1988).

9. F. Freudenstein and E.J. Primrose, J. of Mech., Trans., and Aut. in Des., 106, (1984)

10. J. Spanos and D. Kohli, J. of Mech., Trans., and Aut. in Design, 107, (1985).

11. E.J. Haug, C.M. Luh, F.A. Adkins, and J.Y. Wang, Proceedings of the 23rd ASME Mechanisms Conference, Minneapolis, MN, (1994).

12. M. Ceccarelli and A. Vinciguerra, Int. J. of Rob. Res., 14, No. 2, , (1995).

13. S.K. Agrawal, Int. J. of Robotics and Automation, 7, (1990).

14. G.R. Pennock and D.J. Kassner, ASME J. of Mech. Design, 115, (1993).

15. A. Kumar and K.J. Walsdron, ASME J. of Mech. Design, 103, (1981).

16. D.C.H. Yang and Z.C. Lai, Transactions of ASME, J.of Mech., Transm., and Automation in Design, 107, (1985).

17. J.Y. Wang and J.K. Wu, Mechanics of Structures and Machines, 21, (1993).

18. K. Abdel-Malek and H.J. Yeh, Computer Aided Design,,(1995).

19. I. Vinagradov (in Russian) Mexanika Mashin, 27-28, (1971).

20. K.S. Fu, Gonzalez, and Lee, Robotics: Control, Sensing, Vision, And Intelligence, McGraw-Hill, Inc., New York (1987).

21. E.L. Allgower and K. Georg, Numerical Continuation Methods, Berlin: Springer-Verlag (1990).

22. W.C. Rheinboldt, Numerical Analysis of Parametrized Nonlinear Equations, New York: John Wiley and Sons, (1986).

23. P.J. McKerrow, Introduction to Robotics, Addison Wesley, (1991).

24. M.J. Pratt and A.D. Geisow, The Mathematics of Surfaces, (J. Gregory), Clarendon Press, Oxford, (1986).

25. G. Lucaks, The Mathematics of Surfaces, (J. Gregory), Clarendon Press, Oxford, (1990).

\section{CAPTIONS TO FIGURES}

Figure 1 (a) A serial n-degree-of-freedom manipulator (b) The service sphere and region 
Figure 2 A six degree-of-freedom manipulator

Figure 3 Boundary curve to a service region

Figure 4 (a) Intersection of singular surfaces (b) Partitioning of a surface to sub-surfaces

Figure 5 Singular surfaces (a) $\mathbf{x}^{1}\left(q_{1}^{s_{1}} \mid q_{2}, q_{3}\right)$ due to $q_{1}^{s_{1}}=-\pi / 4, \quad$ (b) $\mathbf{x}^{2}\left(q_{1}^{s_{2}} \mid q_{2}, q_{3}\right)$ due to $q_{1}^{s_{2}}=5 \pi / 4$

Figure 6 Singular surfaces due to (a) $q_{3}^{s_{2}}=0$, (b) $q_{3}^{s_{5}}=\pi$, (c) $q_{2}^{s_{3}}=-\frac{\pi}{4}$, and (d) $q_{2}^{s_{4}}=\frac{\pi}{2}$

Figure 7 Intersection Curves Between $\mathbf{x}^{1}$ and $\mathbf{x}^{2}$ (a) in the $u v$-space, (b) on the surfaces

Figure 8 Intersection curves dividing singular surface $\mathbf{x}^{1}$ into sub-surfaces

Figure 9 Analytical surfaces that are boundary to the workspace

Figure 10 Service regions 


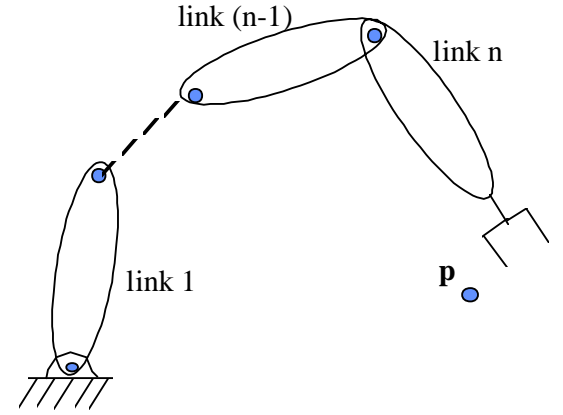

(a)

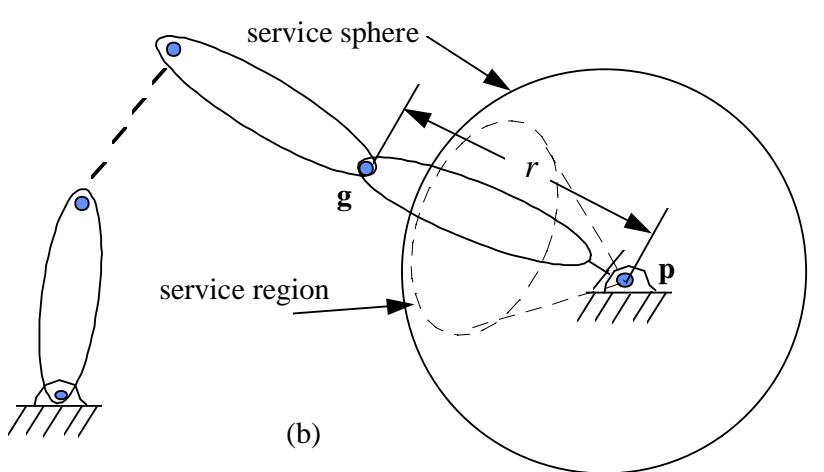

(b)

Fig. 1 (a) A serial n-degree-of-freedom manipulator (b) The service sphere and region 


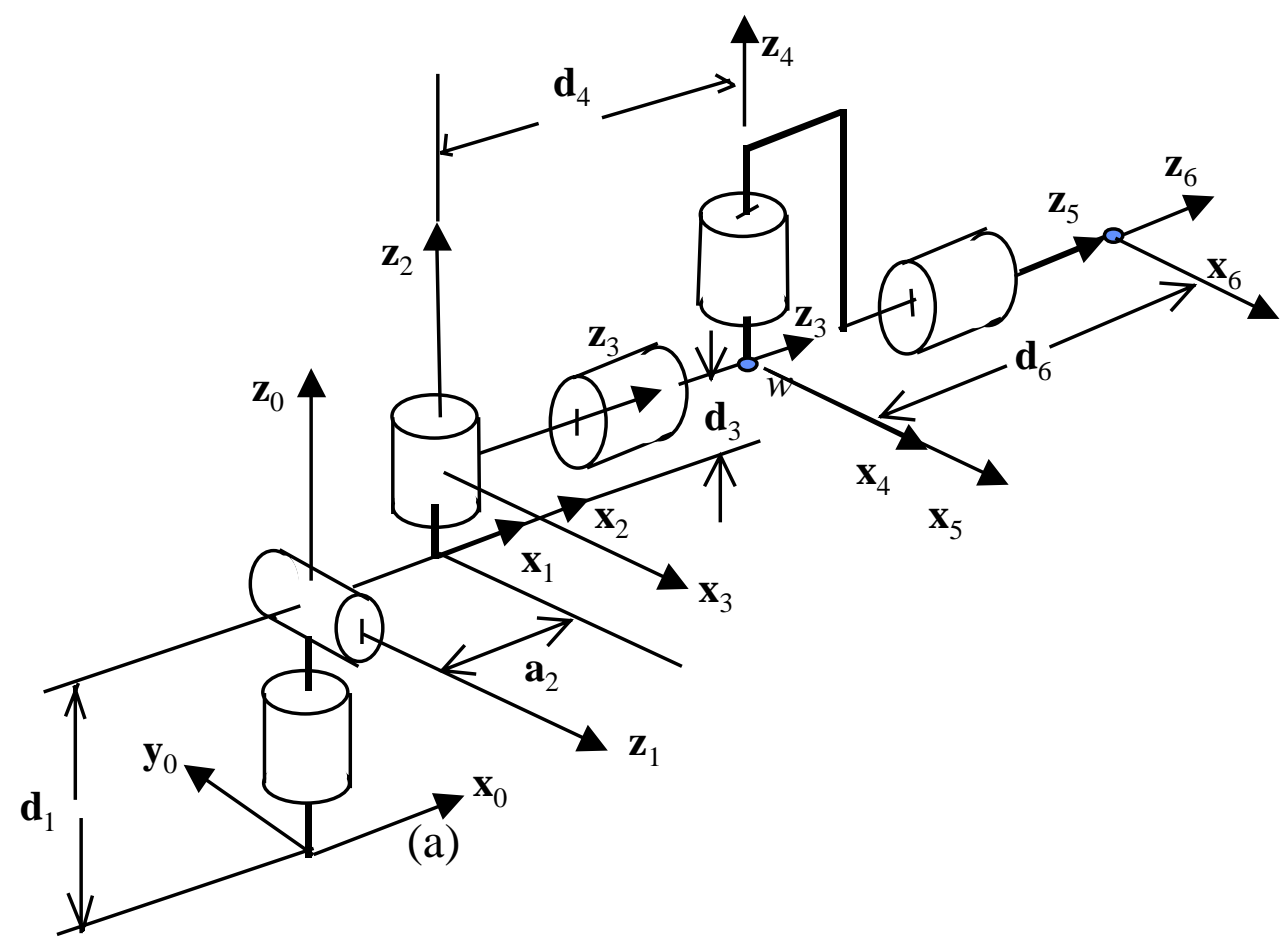

Fig. 2 A six degree-of-freedom manipulator 


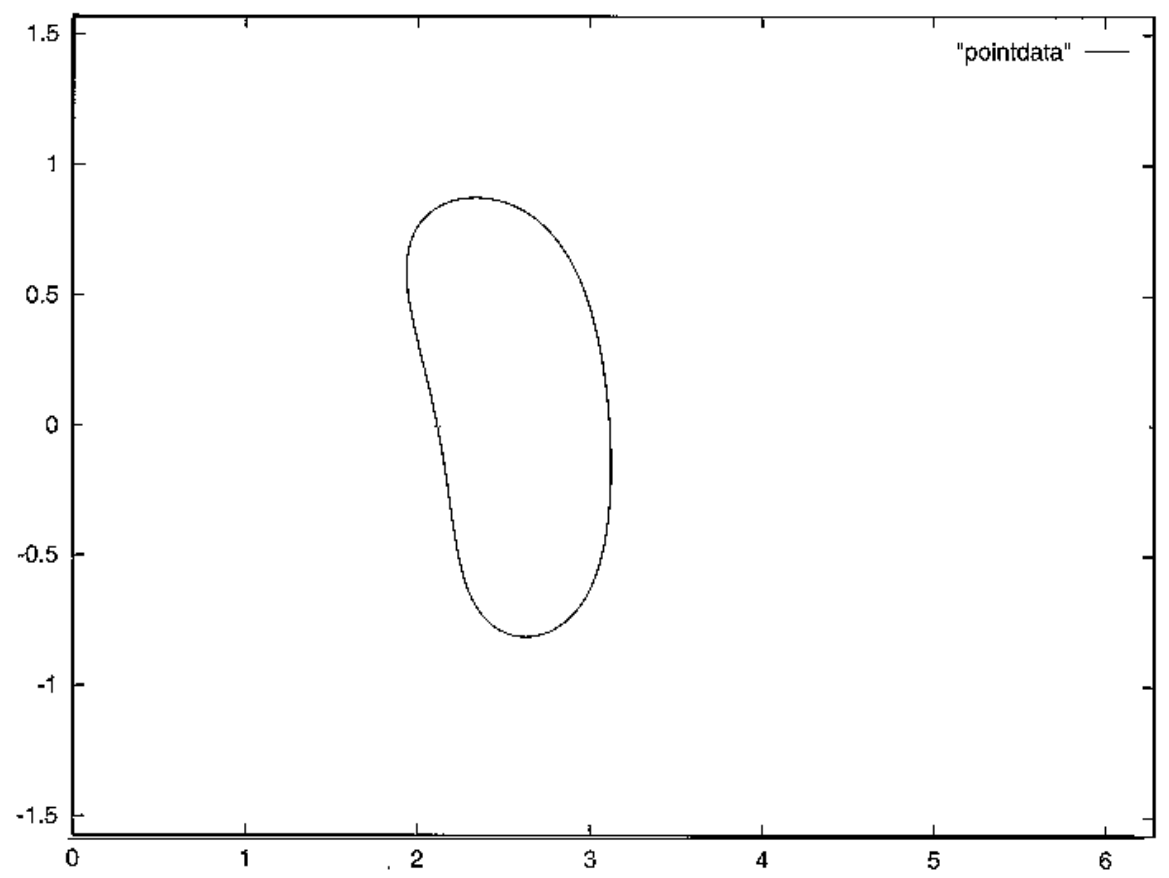

Figure 3 Boundary curve to a service region 


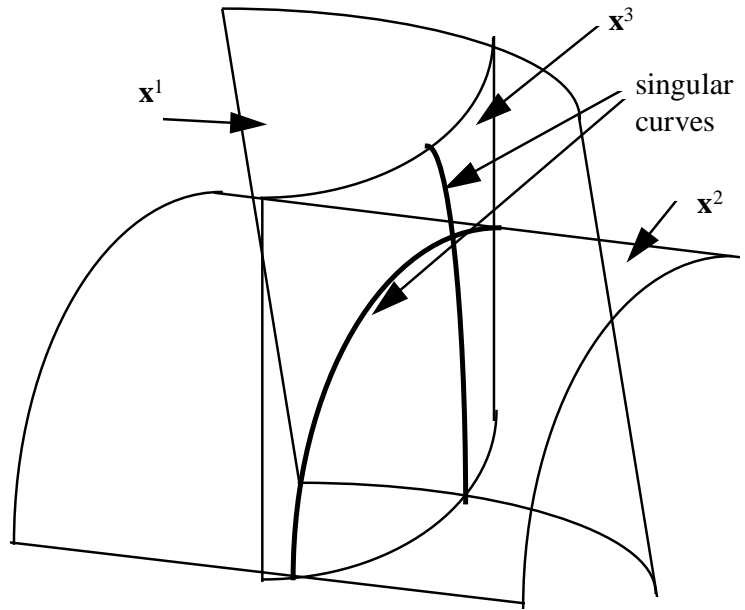

(a)

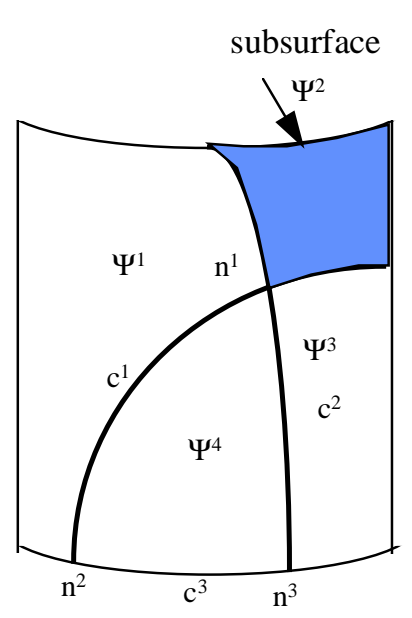

(b)

Fig. 4 (a) Intersection of singular surfaces (b) Partitioning of a surface to sub-surfaces 

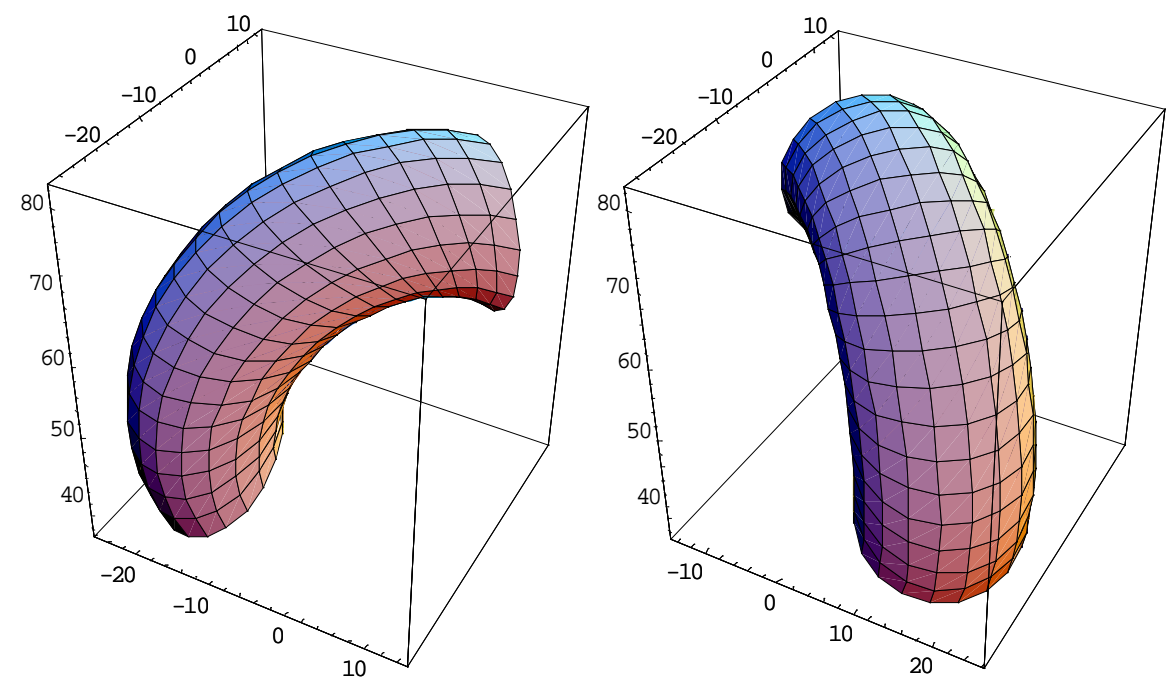

Fig. 5 (a) Singular surfaces (a) $\mathbf{x}^{1}\left(q_{1}^{s_{1}} \mid q_{2}, q_{3}\right)$ due to $q_{1}^{s_{1}}=-\pi / 4$, (b) $\mathbf{x}^{2}\left(q_{1}^{s_{2}} \mid q_{2}, q_{3}\right)$ due to $q_{1}^{s_{2}}=5 \pi / 4$

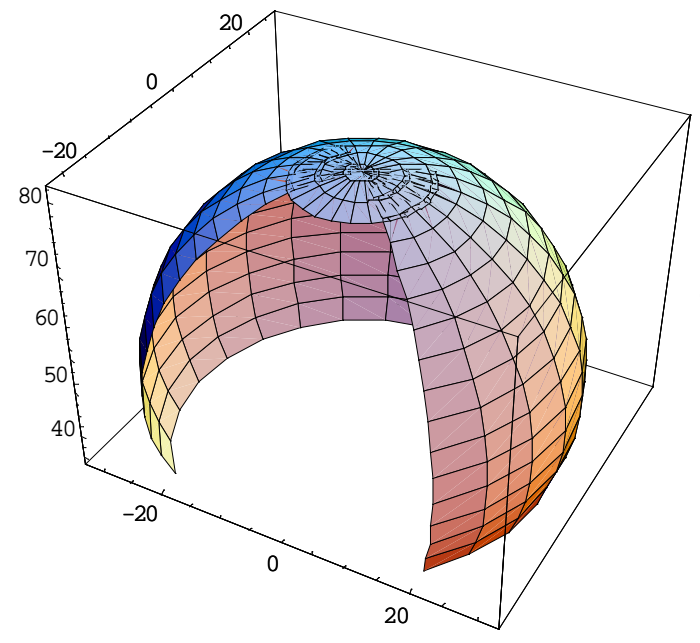

(a)

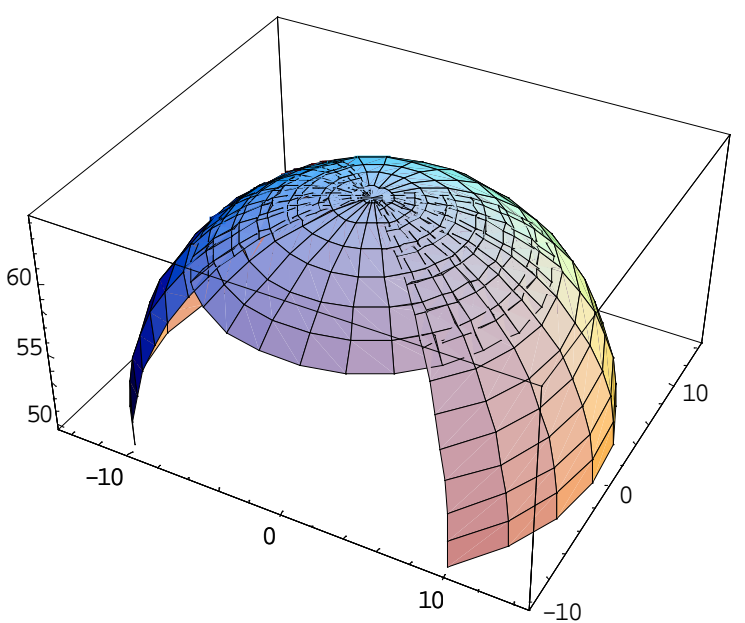

(b)

Fig. 6 (continued) Singular surfaces due to first order singularities 


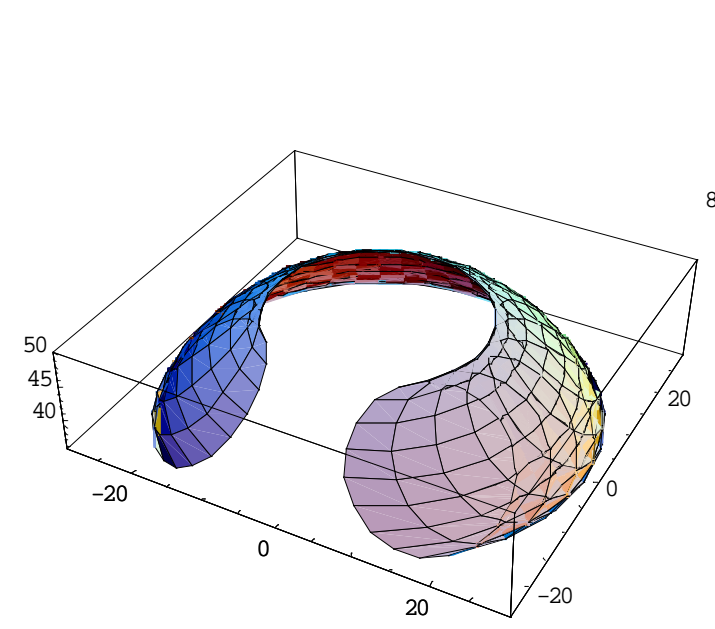

(c)

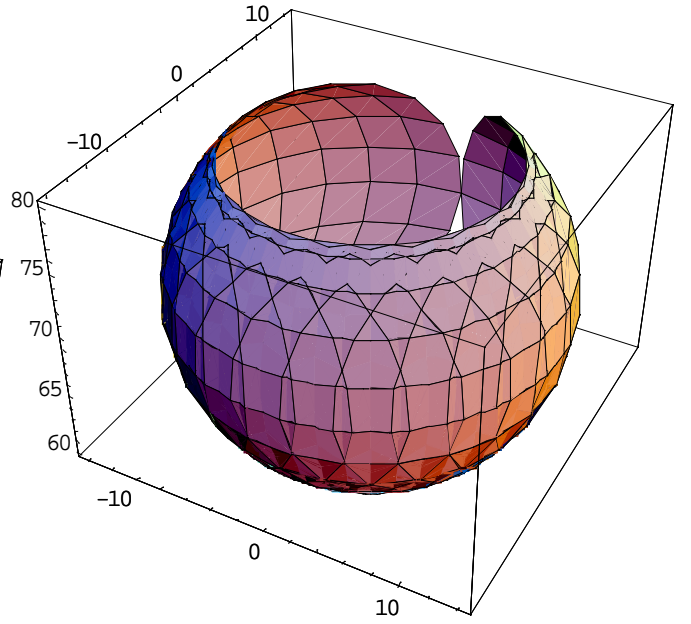

(d)

Fig. 6 (continued) Singular surfaces due to first order singularities
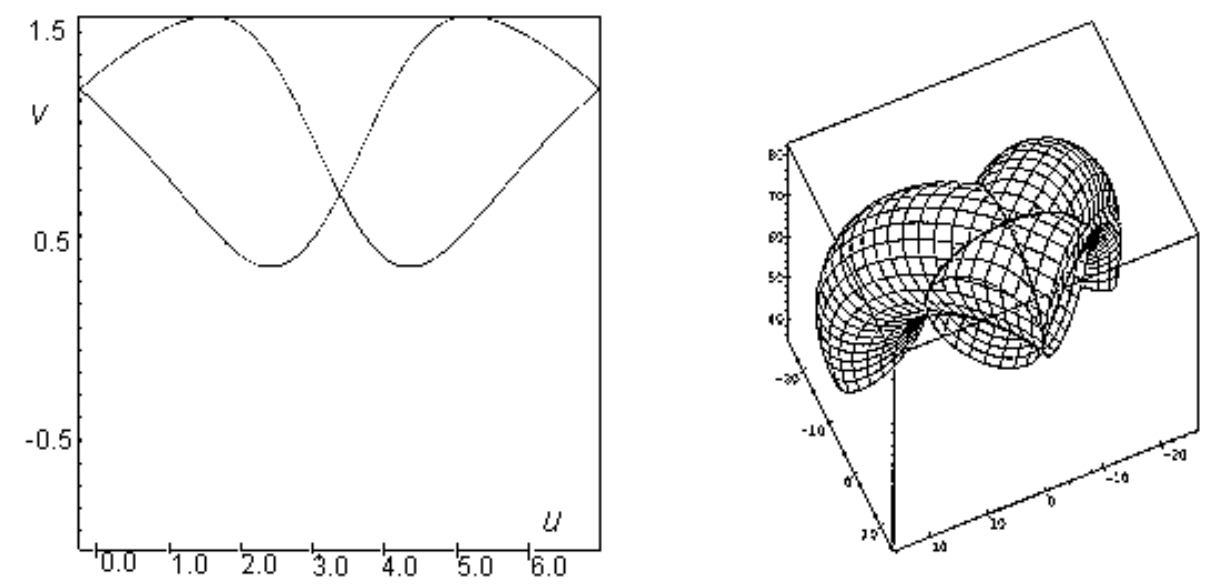

Fig. 7 Intersection Curves Between $\mathbf{x}^{1}$ and $\mathbf{x}^{2}$ (a) in the $u v$-space, (b) on the surfaces 


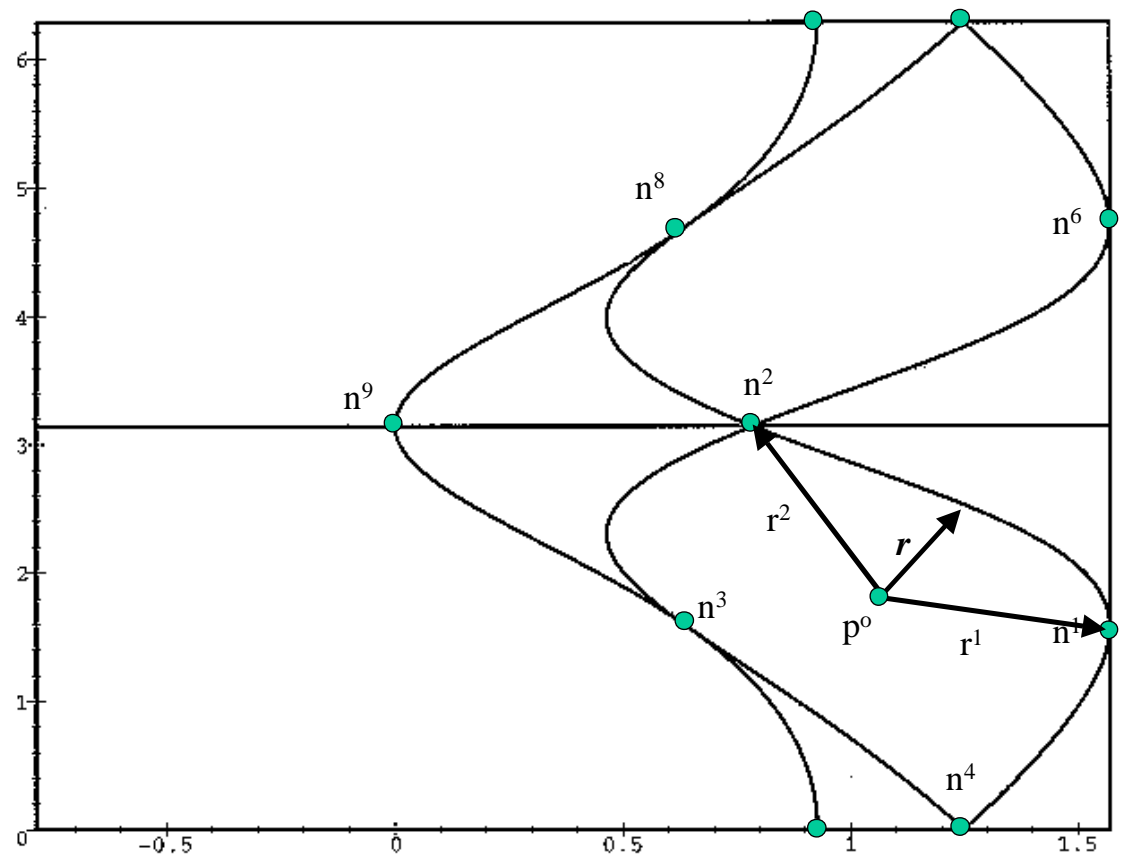

Fig. 8 Intersection curves dividing singular surface $\mathbf{x}^{1}$ into sub-surfaces 


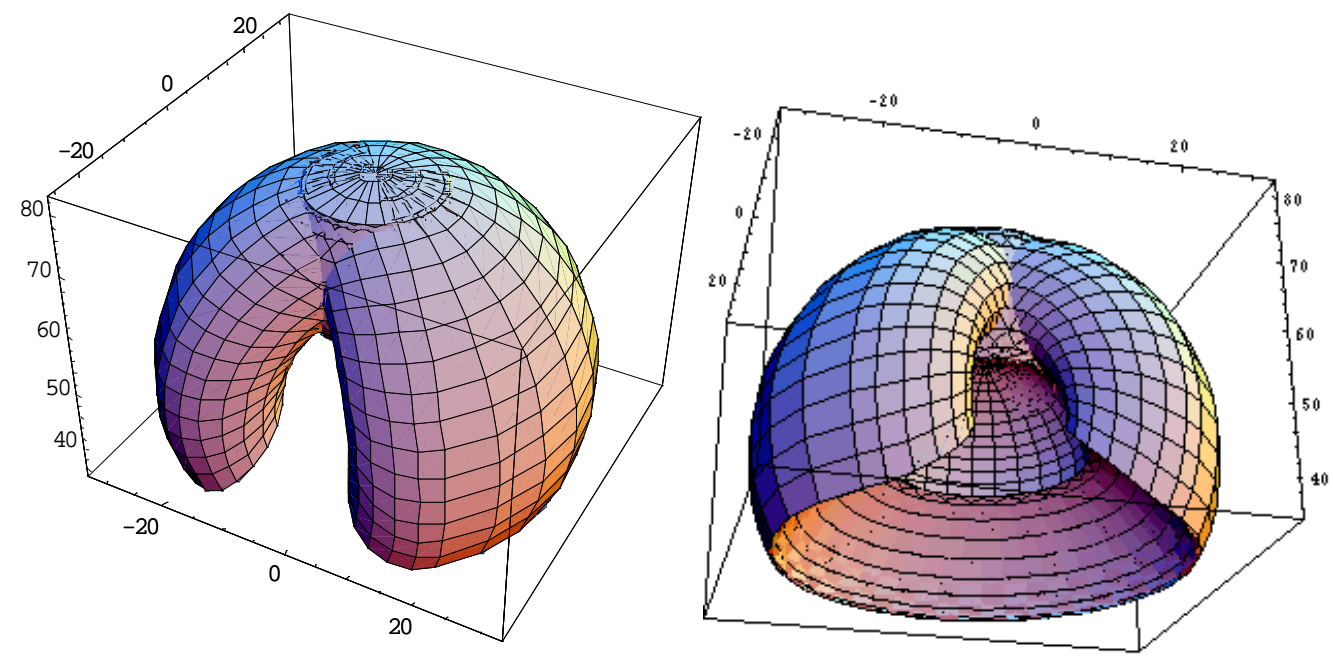

Fig. 9 Analytical surfaces that are boundary to the workspace
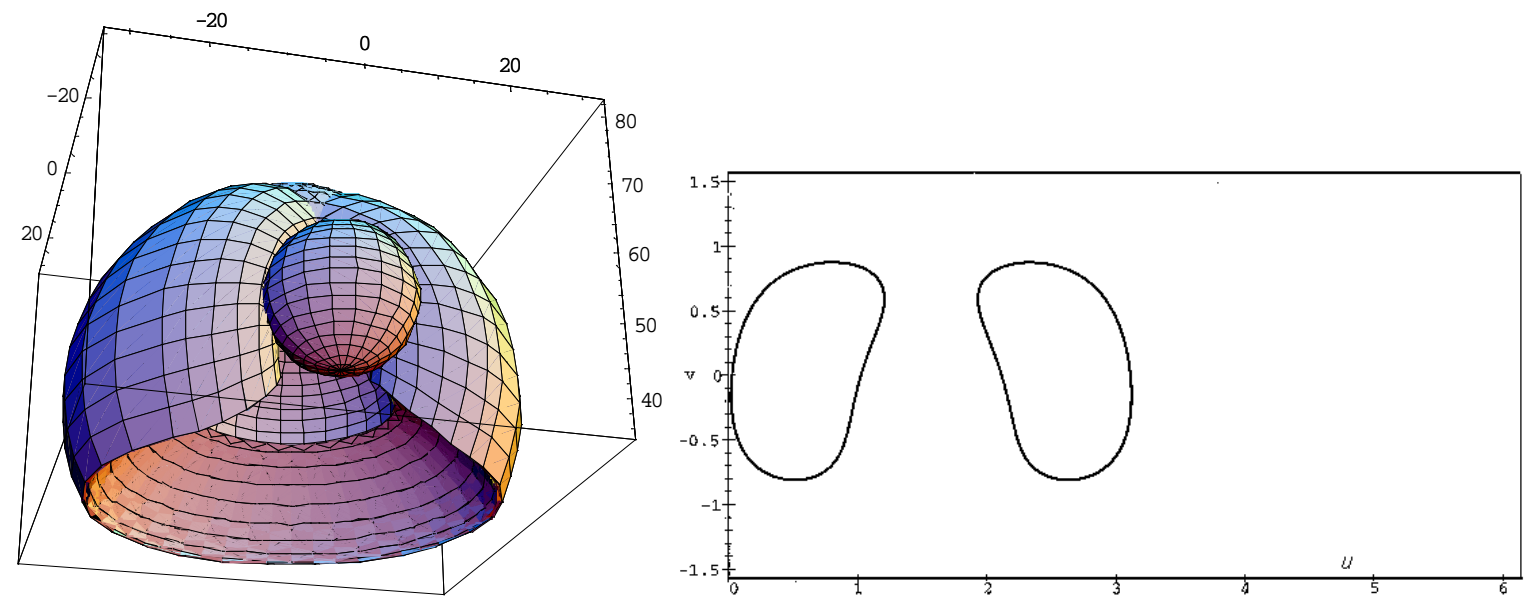

Fig. 10 Service regions 